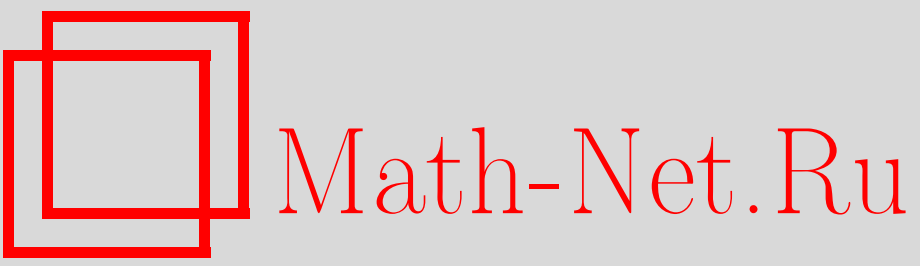

Д. А. Киржниц, Г. В. Шпатаковская, Аномалии в нерелятивистской квантовой механике, ТМФ, 1996, том 109, номер 1, 124-127

DOI: https://doi.org/10.4213/tmf1216

Использование Общероссийского математического портала Math-Net.Ru подразумевает, что вы прочитали и согласны с пользовательским соглашением

http://www . mathnet.ru/rus/agreement

Параметры загрузки:

IP: 3.89 .197 .203

26 апреля 2023 г., 15:44:03 
ТЕОРЕТИЧЕСКАЯ

И МАТЕМАТИЧЕСКАЯ

ФИЗИКА

Том 109, № 1

октябрь, 1996

Д. А. Киржниц* , Г. В. Шпатаковская ${ }^{\dagger}$

\section{АНОМАЛИИ В НЕРЕЛЯТИВИСТСКОЙ КВАНТОВОЙ МЕХАНИКЕ}

Показано, что при достаточной сингулярности потенциала в нерелятивистской системе ферми-частиц возможно появление аномалий (ненулевых значений формально равных нулю величин). Для различных типов сингулярностей (в частности, для кулоновского потенциала) вычислены аномалии, связанные с энергией и полным числом частиц. Такие аномалии полезно использовать при квазиклассическом описании электрон-ядерных систем.

Как известно (см., например, [1]), об аномалиях говорят в ситуации, когда величина, равная нулю в классическом пределе благодаря своим свойствам инвариантности, приобретает конечное значение за счет квантовых эффектов в области высоких энергий (и вообще в области своей сингулярности). Известные примеры аномалий (в том числе исторически первый пример - появление диамагнитной, пропорциональной потенциалу и тем самым нарушающей калибровочную инвариантность компоненты тока в теории поляризации вакуума) относятся к квантовой теории поля. Ниже обсуждаются аномалии в нерелятивистской квантовой механике. Их использование упрощает описание системы взаимодействующих частищ [2].

1. Число частиц $N$ и энергия $E$ ферми-частиц при нулевой температуре $T$ во внешнем поле $U$ даются формулами ${ }^{1)}$

$$
N=2 \operatorname{tr}(\widehat{\rho}), \quad E=2 \operatorname{tr}(\widehat{H} \widehat{\rho}),
$$

где $\widehat{H}=\widehat{p}^{2} / 2 m+U$ - гамильтониан, $\widehat{\rho}=\theta(\mu-\widehat{H})$ - оператор заполнения уровней с верхней границей $\mu(\theta(x)=1$ при $x>0, \theta(x)=0$ при $x<0)$,

$$
\operatorname{tr}(\ldots)=(2 \pi \hbar)^{-3} \int d \vec{x} d \vec{p} \exp (-i \vec{p} \vec{x} / \hbar)(\ldots) \exp (i \vec{p} \vec{x} / \hbar)
$$

\footnotetext{
1) Полученные ниже результаты оказываются справедливыми и при учете взаимодействия между частицами в рамках метода самосогласованного поля (Хартри, Хартри-Фока).
}

* Физический институт им. П.Н.Лебедева РАН

${ }^{\dagger}$ Институт математического моделирования РАН 
При $\mu>0$, чтобы не иметь дела с областями и непрерывного, и дискретного спектров, а также по другим причинам (см. [2, 3]), полезно перейти к выражениям

$$
N=a_{N}-2 \operatorname{tr}(\widehat{\widetilde{\rho}}), \quad E=a_{E}-2 \operatorname{tr}(\widehat{H} \widehat{\widetilde{\rho}}),
$$

где $\widehat{\widetilde{\rho}}=1-\widehat{\rho}$ - оператор заполнения с нижней границей $\mu$,

$$
a_{N}=2 \operatorname{tr}(1), \quad a_{E}=2 \operatorname{tr}(\widehat{H}) .
$$

Непосредственно видно, что зависимость $a_{N}$ и $a_{E}$ от постоянной Планка $\hbar$ сосредоточена в множителе $(2 \pi \hbar)^{-3}$. Поэтому величины $A_{\alpha}=(2 \pi \hbar)^{3} a_{\alpha}, \alpha=N, E$, не зависят от $\hbar$, т.е. инвариантны относительно преобразования

$$
\hbar \rightarrow \hbar+C
$$

Вводя величины $A_{\alpha}^{0}=\lim _{\hbar \rightarrow 0} A_{\alpha}, \alpha=N, E$ (они отвечают модели Томаса-Ферми, где не учитьваются коммутаторы операторов $\widehat{p}^{2} / 2 m$ и $U$ ), заключаем, что

$$
\delta A_{\alpha}=A_{\alpha}-A_{\alpha}^{0}=0, \quad \alpha=N, E .
$$

Эти разности представляют собой полную сумму квантовых и оболочечных поправок к модели Томаса-Ферми [2] для соответствующих величин $A_{\alpha}$.

2. Равенства (3) имеют формальный смысл и могут нарушиться из-за сильной расходимости величин $A_{\alpha}$ и $A_{\alpha}^{0}$ при больших $p$. Такое нарушение действительно происходит, например, в случае достаточно сильной сингулярности $U$ при малых $x$. Соответствующие величины $\delta A_{\alpha}$ и послужат примерами квантово-механических аномалий.

$\mathrm{C}$ целью подавления на промежуточном этапе вклада области больших $p$ введем регуляризацию

$$
\begin{aligned}
& \delta A_{N} \rightarrow \lim _{\Lambda \rightarrow \infty} 2 \Lambda^{2}\left[\left\{\operatorname{Tr}(\Lambda+\widehat{H})^{-2}\right\}-\{\hbar=0\}\right]=-\lim _{\Lambda \rightarrow \infty} 2 \Lambda^{2} \frac{\partial W}{\partial \Lambda}, \\
& \delta A_{E} \rightarrow \lim _{\Lambda \rightarrow \infty} 2 \Lambda^{2}\left[\left\{\operatorname{Tr}\left(\widehat{H}(\Lambda+\widehat{H})^{-2}\right)\right\}-\{\hbar=0\}\right]=-\lim _{\Lambda \rightarrow \infty} 2 \Lambda^{2}\left(1+\Lambda \frac{\partial}{\partial \Lambda}\right) W \\
& \text { где } \operatorname{Tr}=(2 \pi \hbar)^{3} \operatorname{tr} \\
& W=\left[\left\{\operatorname{Tr}(\Lambda+\widehat{H})^{-1}\right\}-\{\hbar=0\}\right]= \\
& =\int d \vec{x} d \vec{p}\left[\left(\Lambda+(\vec{p}-i \hbar \vec{\nabla})^{2} / 2 m+U\right)^{-1}-\left(\Lambda+p^{2} / 2 m+U\right)^{-1}\right],
\end{aligned}
$$

во вторых фигурных скобках в $(4),(5)$ стоят те же величины, что и в первых, но при $\hbar=0$, и градиент в $(5)$ действует на величину $U$.

Делая замену $\vec{p} \rightarrow \Lambda^{1 / 2} \vec{p}, \vec{x} \rightarrow \Lambda^{-1 / 2} \vec{x}, U(\vec{x}) \rightarrow U\left(\Lambda^{-1 / 2} \vec{x}\right)$, видим, что относительный вклад потенциала $U$ в $(5)$ определяется его поведением при $x \rightarrow 0$ (при больших энергиях). Если в этой точке $U$ имеет сингулярность типа $x^{-2}$, то $W \propto \Lambda^{-1}$ и все порядки теории возмущений по $U$ одинаково важны, причем $\delta A_{N}$ имеет конечную величину, а $\delta A_{E}$ согласно (4б) равна нулю (случай А). Если же при малых $x$ потенциал имеет кулоновское поведение, то важны поправки первого $\left(W \propto \Lambda^{-3 / 2}\right)$ и второго $\left(W \propto \Lambda^{-2}\right)$ порядков теории возмушений, причем величина $\delta A_{N}=0$, а $\delta A_{E}$ отлична от нуля (случай Б). Наконец, если особенности $U$ при малых $x$ слабее кулоновской, то важен, самое большее, первый порядок теории возмушений (случай В). 
3. При раскрытии выражения (5) здесь и ниже используется известное разложение

$$
(a+b)^{-1}=a^{-1}-a^{-1} b a^{-1}+a^{-1} b a^{-1} b a^{-1}+\ldots .
$$

Начнем с рассмотрения первого порядка теории возмущений, которое относится ко всем трем упомянутым выше случаям,

$$
W_{1}=-\int d \vec{p} \int d \vec{k} u(\vec{k}) \delta(\vec{k})\left[\left(\Lambda+(\vec{p}+v k)^{2} / 2 m\right)^{-1}-\left(\Lambda+p^{2} / 2 m\right)^{-1}\right]\left(\Lambda+p^{2} / 2 m\right)^{-1} \text {. }
$$

Здесь после усреднения по углам выражение в квадратных скобках при $k \rightarrow 0$ ведет себя как $k^{2}$. Поэтому величина $W_{1}$ отлична от нуля только в том случае, когда $U(k)$ имеет достаточно сильную сингулярность при $k \rightarrow 0$, например вида $\propto k^{-2}$. Это означает, что потенциал $U(x)$ имеет кулоновское поведение $Z e^{2} / x$ при больших $x$. При этом, используя формулу Фейнмана

$$
(a b)^{-1}=\int_{0}^{1} d x[a x+b(1-x)]^{-2},
$$

легко найти

$$
W_{1} \propto(2 \pi \hbar)^{3} Z e^{2} m^{2} /\left[\hbar(2 m \Lambda)^{3 / 2}\right] .
$$

Соответственно $\delta A_{N}=0$, а $\delta A_{E}$ отлична от нуля и бесконечна:

$$
\delta A_{E} \propto(2 \pi \hbar)^{3} Z e^{2}(2 m \Lambda)^{1 / 2} / \hbar .
$$

Этот результат относится к мало интересному с физической точки зрения случаю кулоновского поведения на больших расстояниях (неэкранированная система). Далее мы исключим из рассмотрения этот случай, а также случай более слабого, чем кулоновское, убывания при больших $x$, избежав тем самым необходимость обсуждать первый порядок теории возмущений.

4. Переходим к обсуждению случая $\mathrm{A}\left(U \rightarrow \alpha x^{-2}\right.$ при малых $\left.x\right)$, ограничиваясь вычислением отличной от нуля величины $\delta A_{N}$. Делая замену переменных $\vec{p} \rightarrow \sqrt{2 m \Lambda} \vec{p}$, $\vec{x} \rightarrow \sqrt{\alpha / \Lambda} \vec{x}$, имеем из $(4),(5)$

$\delta A_{N}=-2(2 m \alpha)^{3 / 2} \int d \vec{x} d \vec{p}\left[\left(1+(\vec{p}-i \hbar \vec{\nabla} / \sqrt{2 m \alpha})^{2}+1 / x^{2}\right)^{-1}-\left(1+p^{2}+1 / x^{2}\right)^{-1}\right]$.

Будем считать связь сильной $\left(m \alpha / \hbar^{2} \gg 1\right)$ и ограничимся первым неисчезаюшим членом разложения по $\alpha^{-1}$ :

$$
\delta A_{N}=-\frac{(2 \pi \hbar)^{3}}{36} \sqrt{2 m \alpha} / \hbar .
$$

Подчеркнем, что такая аномалия отражает в определенном смысле нарушение условия полноты системы собственных функций уравнения Шредингера с сингулярным потенциалом $U$. В самом деле, записывая $a_{N}$ в виде

с учетом условия полноты

$$
a_{N}=2 \operatorname{tr}(1)=2 \sum_{\nu} \int d \vec{x} \bar{\psi}_{\nu}(\vec{x}) \psi_{\nu}(\vec{x})=2 \int d \vec{x} \delta(0)
$$

$$
\sum_{\nu} \bar{\psi}_{\nu}(\vec{x}) \psi_{\nu}(\vec{x})=\delta\left(\vec{x}-\vec{x}^{\prime}\right)
$$

мы приходим к формальному выводу о независимости величины $a_{N}=\delta A_{N} /(2 \pi \hbar)^{3}$ от $\hbar$. Наличие расходимостей при высоких энергиях лишает смысла такой формальный вывод (см. (7)). 
5. В этом заключительном разделе обсуждается наиболее интересная с физической точки зрения величина $\delta A_{E}$ для кулоновского поведения $U \propto-Z / r$ на малых расстояниях (случай Б, см. выше). Ограничиваясь, согласно сказанному выше, вторым порядком теории возмушений, имеем

$$
\begin{aligned}
W_{2}= & (2 \pi \hbar)^{-3} \int d \vec{p} \int d \vec{k}|U(\vec{k})|^{2} \times \\
& \times\left[\left(\Lambda+(\vec{p}+\vec{k})^{2} / 2 m\right)^{-1}-\left(\Lambda+p^{2} / 2 m\right)^{-1}\right]\left(\Lambda+p^{2} / 2 m\right)^{-2},
\end{aligned}
$$

где $U(\vec{k})=4 \pi Z e^{2} \hbar^{2} / k^{2}$. Представляя последний сомножитель в (8) в виде

$$
-\left.\frac{\partial}{\partial \Lambda^{\prime}}\left(\Lambda^{\prime}+\frac{p^{2}}{2 m}\right)^{-1}\right|_{\Lambda^{\prime}=\Lambda}
$$

и используя формулу (6), получаем

$$
W_{2}=-(2 \pi \hbar)^{3} \frac{Z^{2}}{8 \Lambda^{2}} \cdot \frac{e^{2}}{a_{0}}
$$

что и ведет согласно (3) к конечному значению

$$
\delta A_{E}=(2 \pi \hbar)^{3} \frac{Z^{2}}{4} \cdot \frac{e^{2}}{a_{0}}
$$

уже приводившемуся без вывода в работе авторов [2].

\section{Список литературы}

[1] Ю.Н.Кафиев. Аномалии и теория струн. Новосибирск: Наука, 1991; А.Ю.Морозов. УФН. 1986. Т. 150. С. 337; У.А.Бардин. УФН. 1986. Т. 150. С. 439.

[2] Д.А.Киржнич, Г.В.Шпатаковская. Письма в ЖЭТФ. 1994. Т. 59. Вып. 7. С. 446; ЖЭЭТФ. 1995. Т. 108. № 4(10). С. 1238.

[3] А.И.Базь, Я.Б.Зельдович, А.М.Переломов. Рассеяние, реакции и распады в нерелятивистской квантовой механике. М.: Наука, 1971.

Поступила в редакцию 3.IV.1996 г.

\section{A. Kirzhnits, G. V. Shpatakovskaya ANOMALIES IN NON-RELATIVISTIC QUANTUM MECHANICS}

The violation of some formal relations (anomalies) for the system of non-relativistic fermi-particles is shown to appear when a singularity of the potential is rather strong. For different singularity types (in part for Coulomb potential) the anomalies associated with the system energy and total number of particles are calculated. These anomalies may be useful in the semiclassical description of electron-nuclear systems. 